\title{
UN TRIPTYQUE BRUGEOIS DE LA RENAISSANCE À CASTROJERIZ (BURGOS) ET LINFLUENCE DE LA MADONE AU CHANOINE VAN DER PAELE EN CASTILLE*
}

\author{
Didier Martens \\ Université Libre de Bruxelles
}

RÉSUMÉ

La Madone au chanoine Van der Paele (Bruges, Groeningemuseum, 1436), I'une des oeuvres les plus célèbres de Jan van Eyck, a suscité depuis la fin du XVème jusqu'au milieu du XVIlème siècle un grand nombre de copies, plus ou moins fidèles, en général réduites au groupe central de la Vierge à l'Enfant. La plupart de ces copies semblent avoir été réalisées à Bruges. C'est certainement le cas des deux triptyques du XVlème siècle portant clairement l'empreinte du prestigieux modèle, qui sont présentés dans cet article. Ils sont conservés aujourd'hui encore dans des églises de la province de Burgos. Le premier, qui présente des liens avec Gerard David, se trouve à San Juan de Castrojeriz, le second, proche d'Ambrosius Benson, à San Martín Obispo de Santa Cruz de Juarros. Si la référence à la Madone au chanoine Van der Paele est manifeste, il apparaît toutefois que les auteurs des deux triptyques n'ont pas pris pour exemple l'original eyckien lui-même, mais un modèle intermédiaire. Par ailleurs, l'auteur du triptyque de Castrojeriz s'est permis d'opérer une véritable critique en acte du groupe marial imaginé par Jan van Eyck. Les deux oeuvres non seulement jettent un éclairage sur la pratique de la copie d'après les grands maîtres à Bruges au XVlème siècle, elles mettent également en évidence l'intérêt que suscitaient de telles images 'rétrospectives' dans la Castille contemporaine.

Paroles-clés: Ambrosius Benson, Brujas, Copia, Gerard David, Jan van Eyck

\section{ABSTRACT}

One of Jan van Eyck's most celebrated works, Virgin and Child with Canon Van der Paele (Bruges, Groeningemuseum, 1436) inspired a large number of copies between the late 15th and mid-17th centuries. These copies, which varied in their faithfulness to the original, generally took as their subject the central group formed by the Virgin and Child, and most of them seem to have been made in Bruges. This is certainly the case of the two 16thcentury triptychs discussed in this article, which remain housed in churches in the province of Burgos and clearly bear the imprint of the much-lauded work on which they are based. The first, which has links with Gerard David, can be found in the church of San Juan in Castrojeriz, and the second, close to Ambrosius Benson, in San Martín Obispo in Santa Cruz de Juarros. While the reference to Virgin and Child with Canon Van der Paele is obvious, it nevertheless appears to be the case that the authors of the two triptychs used an intermediate work as their model rather than the original painting. Furthermore, the author of the triptych held in Castrojeriz offered up a genuine critique of the composition, as imaginated by Van Eyck. Not only do the two works shed light on the practice of copying the works of the great masters in 16th century Bruges, they also reveal the interest aroused by such "retrospective" images in contemporary Castile.

Keywords: Ambrosius Benson, Bruges, copy, Gerard David, Jan van Eyck. 
I.

Si la localité castillane de Castrojeriz, située à quelque cinquante kilomètres à l'ouest de Burgos, compte aujourd'hui moins de neuf cents habitants et paraît déserte durant la période hivernale, il n'en a pas toujours été ainsi. Sur la base de documents fiscaux contemporains, sa population peut être estimée, pour l'année 1563, à plus de 4.500 âmes $^{1}$. L'époque d'apogée démographique correspond aux $X V^{e}$ et $X V l^{e}$ siècles. La ville, traversée par le chemin de saint Jacques, était alors en communication avec l'Europe entière et constituait un centre économique important. De riches marchands s'étaient établis, donnant naissance à de véritables dynasties. Ils devaient leur fortune principalement au commerce lainier. Depuis l'époque des Rois Catholiques, la laine castillane, d'excellente qualité, était exportée, en particulier vers Bruges, où elle alimentait l'industrie drapière. C'est ce commerce florissant avec les Flandres qui permit aux marchands de Castrojeriz d'avoir accès durant tout le XVle siècle à des objets de prestige particulièrement prisés dans le sud de l'Europe: les tableaux flamands. Ceux-ci constituaient de véritables status symbols, rendant visible, par leur perfection technique et leur réalisme sidérant, la position sociale prééminente de leur commanditaire ou de leur acheteur.

De nombreuses peintures flamandes demeurent aujourd'hui à Castrojeriz. Parmi ces vestiges de l'époque où la ville comptait dans l'économie européenne, il faut mentionner les douze panneaux d'un retable qui se trouve encore in situ, dans la niche même pour laquelle il fut créé, en I'église Saint-Jean² (fig. 1). Encadrement de bois compris, l'ensemble mesure plus de quatre mètres de hauteur. Comme l'indiquent les armoiries apposées sur le couronnement, il fut commandé par une famille de marchands de Castrojeriz, les Gallo, dont une branche s'était installée à Bruges. C'est dans cette ville qu'il fut peint, probablement dans la quatrième décennie du $\mathrm{XVl}$ e siècle, par un disciple d'Ambrosius Benson que I'on peut dénommer le 'Maître de Castrojeriz'. La commande est singulière. En effet, le retable, composé de quatre séries horizontales de panneaux agencés en trois colonnes, est de type ibérique. Le peintre brugeois a donc réa- lisé, à l'intention des Gallo, un ensemble qui, par le style et la technique, relève clairement de la peinture flamande mais qui, du point de vue typologique, ressortit à la tradition espagnole et portugaise des grands retablos muraux à multiples panneaux superposés.

\section{II.}

À la différence du retable des Gallo, le triptyque actuellement en dépôt à l'église Saint-Jean de Castrojeriz et qui proviendrait de la collégiale Nuestra Señora del Manzano est une œuvre flamande on ne peut plus classique qui, ni par sa forme ni par son format, n'aurait déparé dans

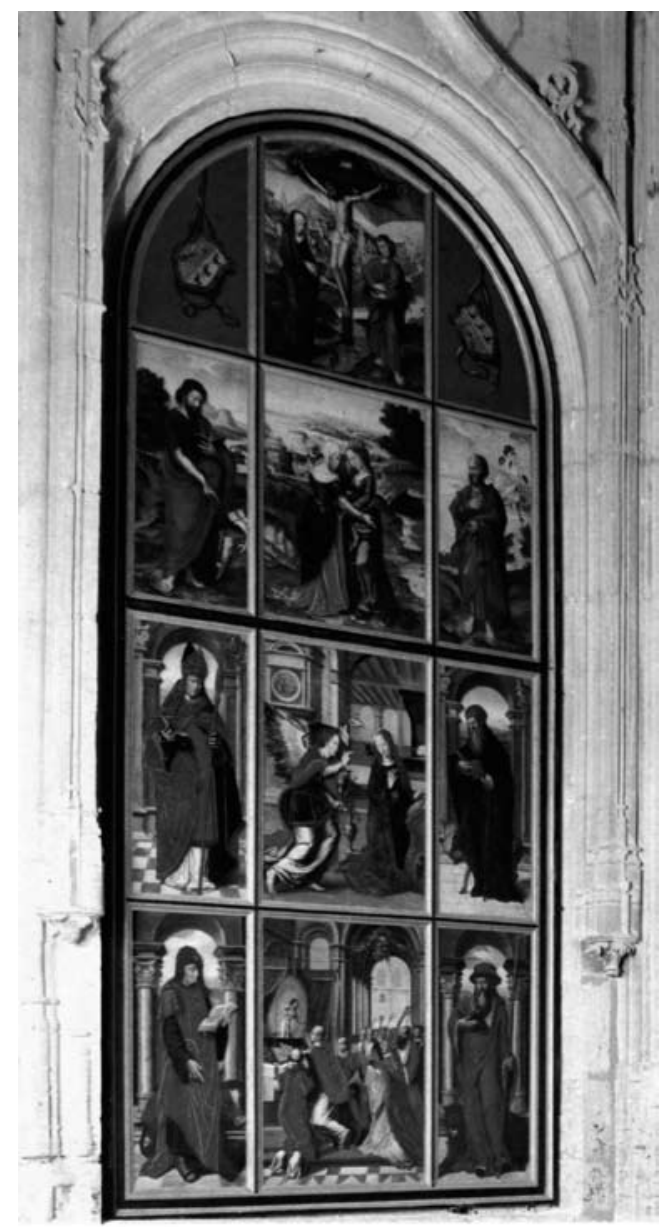

Fig. 1. Maître de Castrojeriz: Retable des Gallo. Castrojeriz, San Juan (photo Alfredo Presencio, Castrojeriz) 

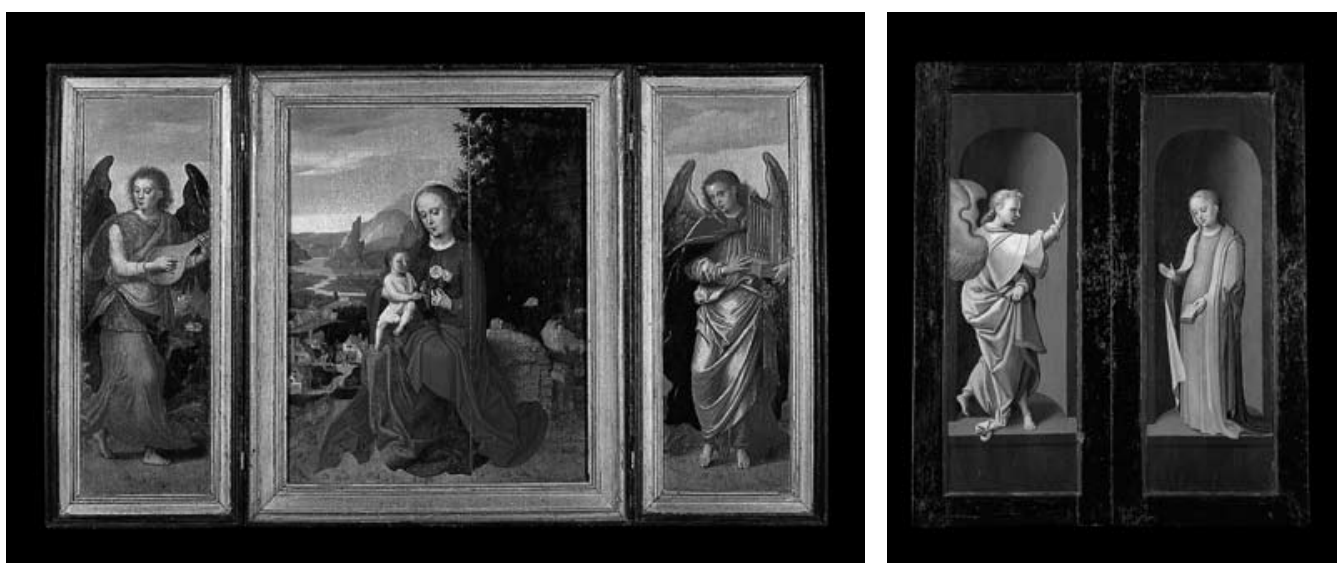

Fig. 2. Anonyme brugeois (XVle siècle): Triptyque de la Madone au perroquet. Castrojeriz, San Juan (photo Alfredo Presencio, Castrojeriz)

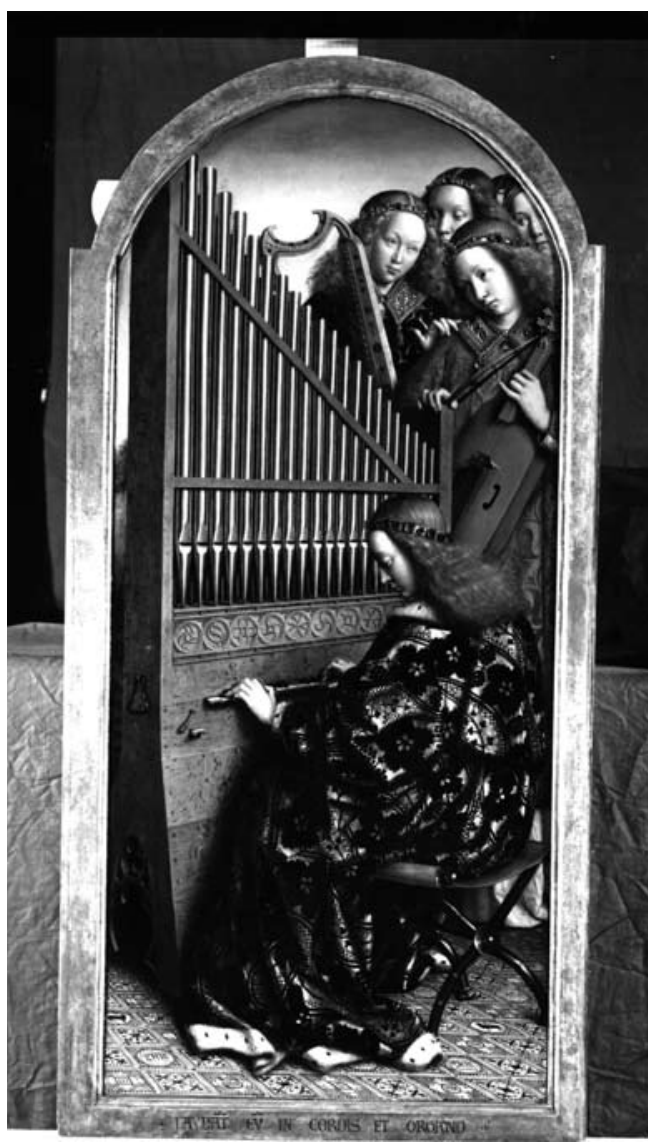

Fig. 3. Jan van Eyck: Polyptyque de l'Agneau mystique, Anges musiciens. Gand, Sint-Baafskathedraal (photo KIKIRPA, Bruxelles) une église de Bruges ${ }^{3}$ (fig. 2). Elle atteste le goût des élites sociales castillanes pour ce que I'on peut appeler l'"exotisme flamand intégral', par opposition à l'exotisme flamand mitigé' du retable des Gallo ${ }^{4}$. Au revers des volets figure I'Annonciation, représentée sous la forme de deux statues blanches insérées dans des niches de couleur rose. Gabriel se trouve à main gauche, Marie à droite. On relèvera une anomalie dans l'éclairage: la paroi dans laquelle les niches ont été aménagées semble curieusement se trouver à contre-jour, alors que les niches elles-mêmes sont éclairées par une source de lumière située en avant, sur la gauche.

Traditionnellement, I'Annonciation occupe le revers des volets de retables, dans les Flandres comme dans l'Empire germanique. De la même façon qu'elle ouvre I'histoire du Salut dans I'Évangile de Luc (I, 26-38), elle sert d'introduction au programme figuré des triptyques. Ce thème est attesté dès les années 1400 au revers des volets d'un petit triptyque portatif franco-flamand en cuivre doré appartenant au Musée Historique de Francfort-sur-le-Main ${ }^{5}$. Deux siècles plus tard, on retrouve encore le même thème chez Rubens, au revers des volets du triptyque de la Lapidation de saint Étienne, conservé au Musée des Beaux-Arts de Valenciennes ${ }^{6}$. Quant à la formule consistant à représenter l'Annonciation sous la forme de deux statues non polychromées, elle est attestée depuis l'époque de Jan van Eyck, notamment sur le triptyque de Dresde qu'il signa en $1437^{7}$. 
Quand le triptyque de Castrojeriz est ouvert, on aperçoit une représentation de la Vierge à I'Enfant dans un paysage. Sur l'avers des volets figurent deux anges musiciens. Celui de gauche joue d'un luth, celui de droite d'un orgue portatifi. Ensemble, ils évoquent un verset des Psaumes $(\mathrm{CL}, 4)$ : «Laudate eum in chordis et organo». Dans la peinture flamande, ce psaume avait déjà été illustré par les frères Van Eyck sur un panneau de l'Agneau mystique: celui des $A n$ ges musiciens (fig. 3 ). Le texte vétéro-testamentaire est d'ailleurs retranscrit sur le talus du cadre de ce panneau.

Dans l'agencement des deux figures d'anges du triptyque de Castrojeriz, on a tenu compte du sens traditionnel de lecture en vigueur dans l'art occidental: de la gauche vers la droite. Le Psalmiste mentionne d'abord les instruments à cordes, puis l'orgue. C'est pourquoi le luthiste ailé occupe le volet gauche, précèdant ainsi, dans le 'texte pictural', l'organiste du volet droit. Tous deux montrent au spectateur comment rendre hommage à Dieu «in chordis et organo». Le «eum» du psaume, c'est l'Enfant Jésus du panneau central.

La Mère de Dieu est représentée deux fois: à I'extérieur, sur le volet droit, et à l'intérieur, sur le panneau central. Par la simple ouverture du triptyque, la sculpture non polychromée du revers semble s'animer, devenir vivante. La Vierge en calcaire blanc de l'Annonciation paraît se transformer, sous les yeux du spectateur, en une Vierge dotée des couleurs de la vie. Le lien qui unit les deux figures est aisément reconnaissable: elles ont le même visage rond, vu de trois-quarts et légèrement incliné vers la gauche. Le peintre a fait en sorte que le spectateur ait l'impression qu'il s'agit bien de la même personne.

Pris dans sa totalité, le triptyque de Castrojeriz constitue donc un dispositif complexe qui, en faisant voir tout d'abord l'image peinte d'une image sculptée de la Vierge -une représentation au second degré-, puis une simple image peinte -une représentation au premier degré-, confère à cette dernière un supplément inattendu de 'présence'. On notera que les frères Van Eyck avaient déjà fait usage d'une telle formule dans le retable de l'Agneau mystique. Au revers du volet gauche figure, dans une niche, une effigie sculptée, non polychromée, du Précurseur. Lorsque le polyptyque est ouvert, on aperçoit à nouveau saint Jean-Baptiste mais, cette fois, il porte un manteau vert et exhibe des joues rouges. L'étonnant Diptyque Van de Velde d'Adriaen Isenbrant, aujourd'hui partagé entre l'église No-

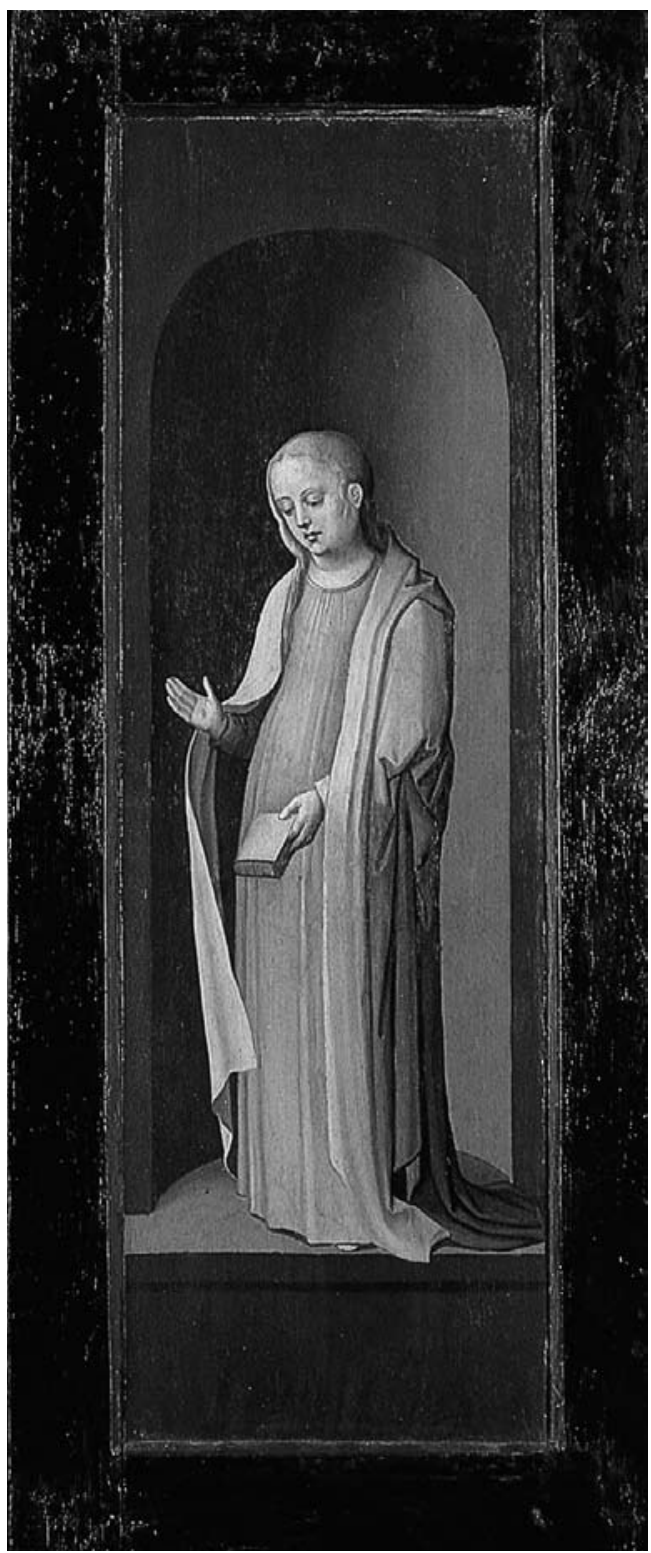

Fig. 4. Anonyme brugeois (XV|e siècle): Triptyque de la Madone au perroquet, Vierge de I'Annonciation. Castrojeriz, San Juan (photo Alfredo Presencio, Castrojeriz) 
tre-Dame de Bruges et les Musées royaux des Beaux-Arts de Belgique à Bruxelles, comporte un autre exemple particulièrement spectaculaire de cet effet d'animation'. Quand on ouvre ce diptyque, on substitue à une représentation sculptée de la Vierge des Sept Douleurs une autre Vierge des Sept Douleurs, pourvue cette fois des couleurs naturelles.

\section{III.}

Le triptyque de Castrojeriz fut présenté pour la première fois au grand public en 1921 à Burgos, lors de l'exposition d'art ancien organisée à I'occasion du $700^{\mathrm{e}}$ anniversaire de la pose de la première pierre de la cathédrale gothique. Dans le catalogue de cette manifestation, publié cinq ans plus tard, l'œuvre est mise en relation avec le peintre brugeois Gérard David: on précise que le panneau central serait la «copie d'un Gérard David perdu» ${ }^{10}$. En 1999, René-Jesús Payo Hernanz reprit ce nom à son compte dans le cata-

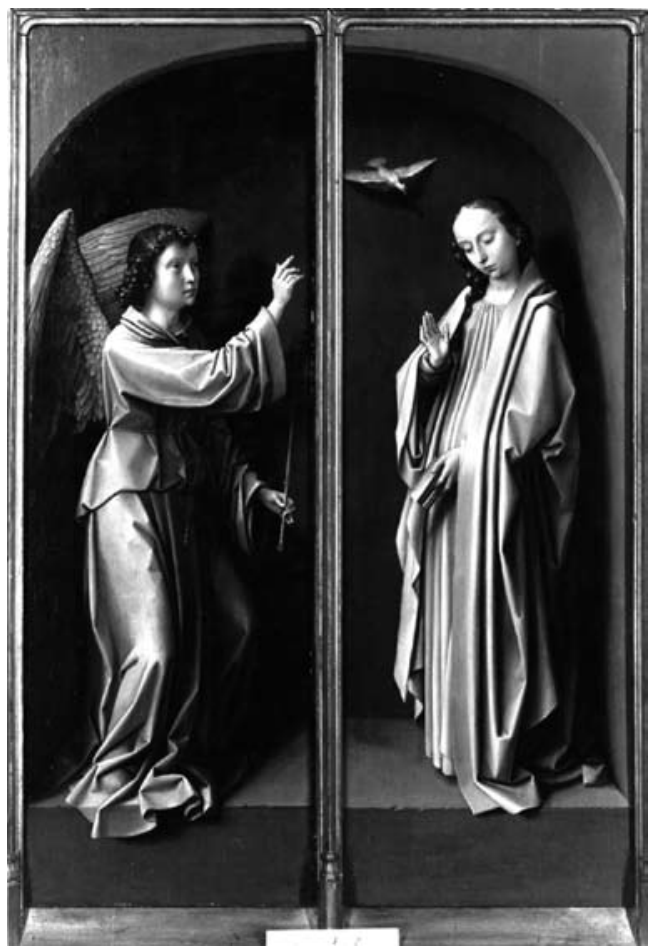

Fig. 5. Gerard David: Triptyque de la Passion, Annonciation. New York, The Metropolitan Museum of Art (photo musée) logue-inventaire des peintures de Castrojeriz. Il propose d'attribuer l'ensemble à un «anonyme flamand», «disciple de Gérard David» et avance une datation «vers 1530-1540»"11. De toute évidence, le triptyque a été peint à Bruges. II procède de différents modèles qui accréditent

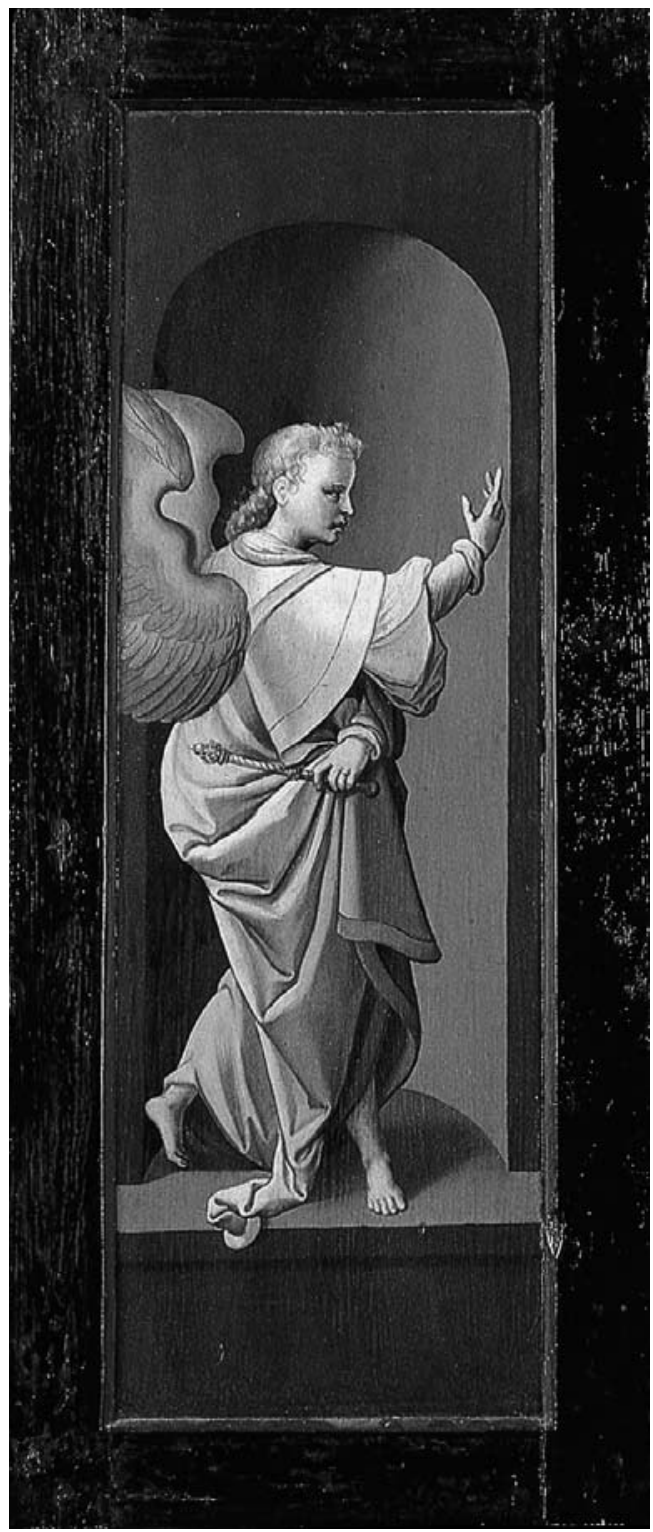

Fig. 6. Anonyme brugeois (XVI e siècle): Triptyque de la Madone au perroquet, Archange de l'Annonciation. Castrojeriz, San Juan (photo Alfredo Presencio, Castrojeriz) 
I'hypothèse d'une origine brugeoise. Ils sont en effet associés à des peintures produites dans la cité flandrienne.

Dans l'Annonciation, deux de ces modèles peuvent être identifiés. Comme l'a remarqué René-Jesús Payo, la figure de Marie (fig. 4) a été empruntée à David ${ }^{12}$. Une Vierge de I'Annonciation quasi semblable, en semi-grisaille sur fond gris, se trouve au revers du volet droit du triptyque de la Passion ${ }^{13}$ (fig. 5). Aujourd'hui partagé entre New York et Philadelphie, il est situé par Maryan Ainsworth vers 1505. Le triptyque de Castrojeriz remonte sans doute déjà au deuxième quart du XVle siècle. Bien qu'il soit reproduit fidèlement, le modèle davidien a été mis au goût du jour. Sous l'influence de l'art classique transmis par I'Italie, la figure mariale est devenue moins anguleuse et plus charnelle, le visage s'est arrondi, les plis de la robe sont cassés et creusés de manière moins brutale. En outre, le drapé a été simplifié, le spectateur comprend plus facilement la structure du manteau du côté gauche.

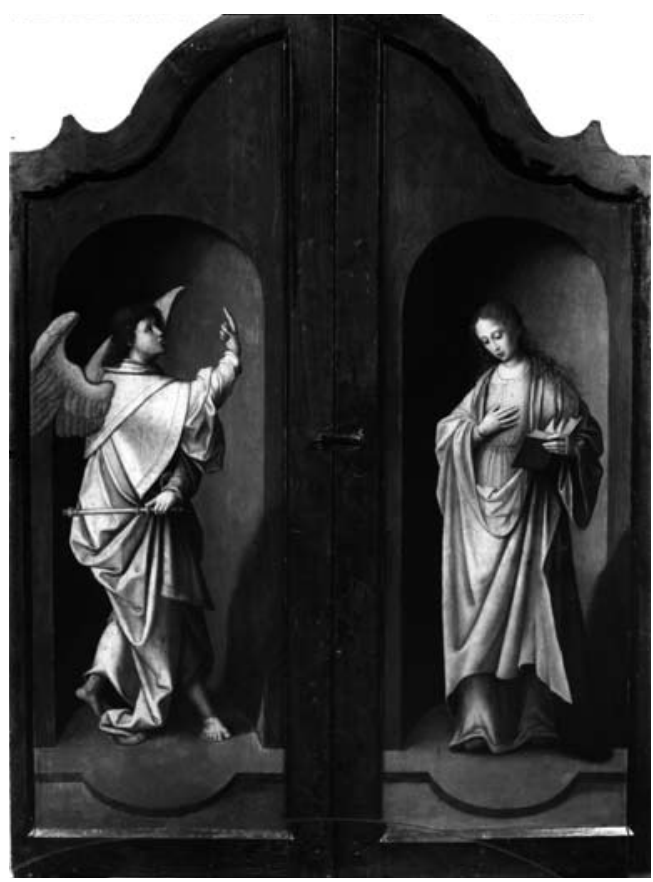

Fig. 7. Entourage d'Ambrosius Benson: Triptyque de saint Jacques matamoros, Annonciation. Anvers, Museum Mayer Van den Bergh (photo KIKIRPA, Bruxelles)

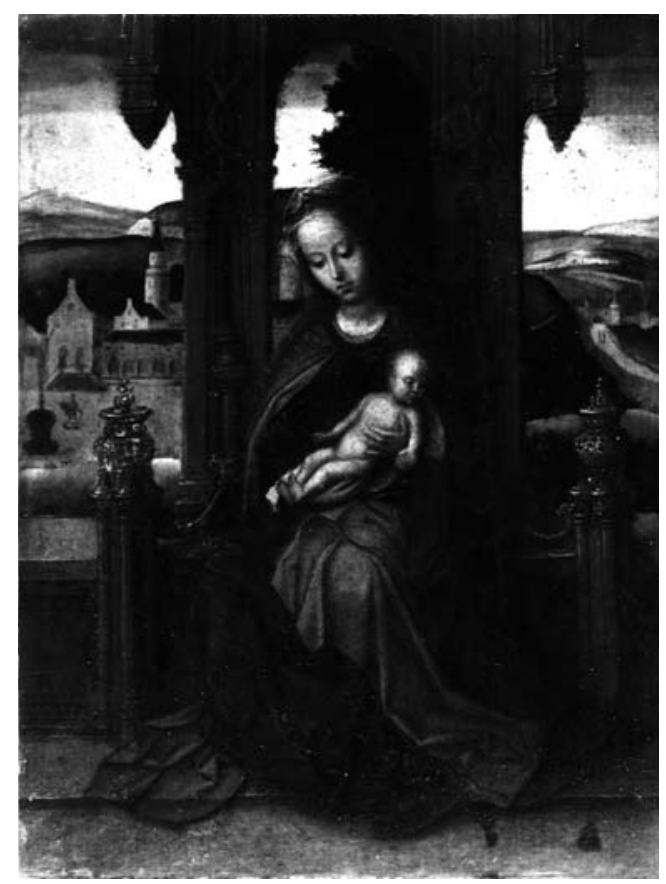

Fig. 9. Entourage d'Adriaen Isenbrant: Madone trônant. Palerme, Galleria nazionale della Sicilia (photo KIKIRPA, Bruxelles) 
Gabriel (fig. 6) dérive d'un autre modèle, sans doute plus récent que la Vierge de David. Un archange similaire peut être identifié au revers du volet gauche d'un triptyque conservé au Musée Mayer Van den Bergh d'Anvers (fig. 7). Attribué par Georges Marlier à Ambrosius Benson, il représente, sur le panneau central, Saint Jacques matamoros $^{14}$. Le choix d'un tel sujet suggère que ce triptyque était, lui aussi, destiné à un client espagnol. Au revers des volets figure une Annonciation en semi-grisaille sur fond gris.

Les deux archanges frappent par leur attitude complexe. Le bras droit est levé, le gauche abaissé. Le sceptre, tenu à I'horizontale devant le corps, est curieusement dirigé vers l'arrière. Au chiasme des bras répond celui des jambes: le pied droit est avancé, le gauche rejeté vers I'arrière. Par rapport à la figure contorsionnée visible sur le triptyque du Musée Mayer Van den Bergh, le messager ailé du triptyque de Castro- jeriz produit une impression plus apaisée. Est-ce également la conséquence de l'influence classique déjà détectée dans la figure mariale? En tout cas, la tête de l'archange n'est pas ramenée vers l'arrière ni légèrement tournée vers l'extérieur, et ses ailes ne sont pas écartées, comme dans le triptyque d'Anvers. Dans celui de Castrojeriz, visage et ailes s'inscrivent dans le plan du corps vu de profil.

Sur le panneau central (fig. 8), deux autres modèles ont été combinés. Marie porte un manteau rouge qui, en retombant sur le genou gauche, donne naissance à une cascade de plis. Ces plis se retrouvent sous une forme très semblable dans une Vierge à l'Enfant flamande, connue par une série d'exemplaires conservés notamment au Musée du Prado à Madrid, à la Galerie Régionale de Palerme (fig. 9), au Musée Boymans-Van Beuningen de Rotterdam et au Museum of Art de San Diego ${ }^{15}$. Toutes ces œuvres ont été attri-

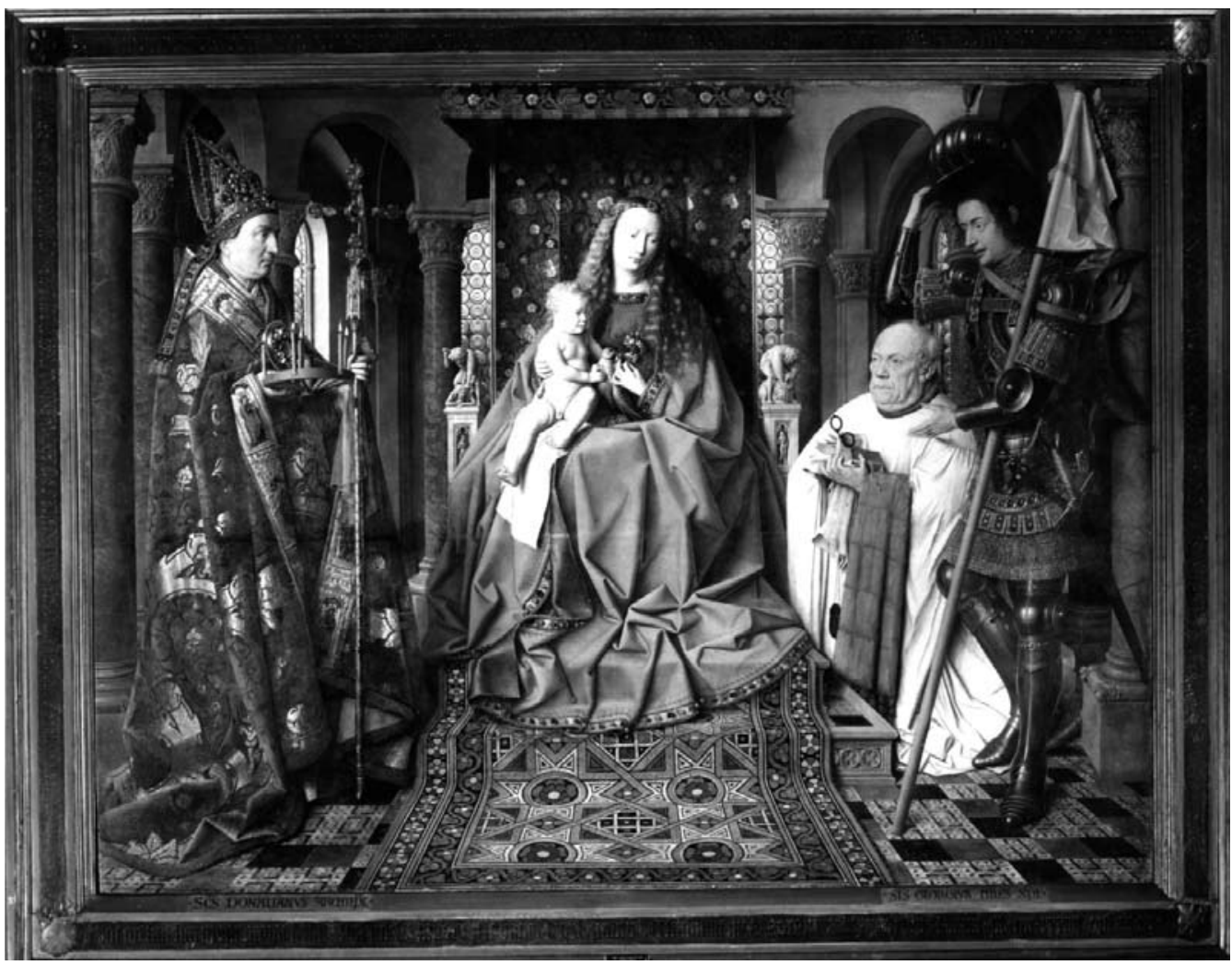

Fig. 10. Jan van Eyck: Madone au chanoine Van der Paele. Bruges, Groeningemuseum (photo KIKIRPA, Bruxelles) 
buées à Isenbrant, ce qui rend vraisemblable une origine brugeoise.

C'est également à un modèle brugeois que l'auteur du panneau central du triptyque de Castrojeriz a emprunté l'Enfant Jésus, le perroquet et la main gauche de Marie. Ces différents motifs proviennent en effet, comme l'a fait observer pour la première fois le chanoine Agustín Lázaro López ${ }^{16}$, de la fameuse Madone au chanoine Van der Paele du Musée Groeninge de Bruges, signée en 1436 par Jan van Eyck ${ }^{17}$ (fig. 10). Ils ont été reproduits fidèlement. Seule adaptation notable, Jésus est revêtu d'une chemise. II ne s'agit nullement d'un surpeint de pudeur moderne, comme on pourrait être tenté de le croire de prime abord. Les parties génitales de l'Enfant sont d'ailleurs visibles. La photographie du panneau central dans l'infrarouge a montré que la chemise était prévue dès le stade du dessin sous-jacent (fig. 11). On se rappellera ici que la Madone au chanoine Van der Paele est une scène d'intérieur, située dans une architecture romane. En installant au premier plan d'un paysage I'Enfant eyckien, I'auteur du triptyque aura sans doute estimé nécessaire de le couvrir.

Le triptyque de Castrojeriz est-il bien l'œuvre d'un seul et unique 'anonyme flamand'? L'hypothèse d'une collaboration, déjà envisagée par Agustín Lázaro et René-Jesús Payo ${ }^{18}$, mérite un examen. La photographie du triptyque ouvert dans l'infrarouge a livré des résultats contradictoires. Dans le panneau central, on observe un dessin sous-jacent peu abondant, dominé par des droites. Le drapé de la Vierge a été mis en place par quelques traits éparts, assez épais, parfois doublés (fig. 12). Ce dessin raide et sommaire contraste nettement avec celui, beaucoup plus souple et plus élaboré, que l'on décèle sur les faces des volets. Là, la photographie dans l'infrarouge a mis en évidence un jeu complexe d'arabesques tracées d'une main légère (fig. 13). Les lignes sont nombreuses. Le dessin sousjacent à l'Annonciation n'a malheureusement pas pu être étudié.

L'examen des figures peintes semble confirmer l'hypothèse d'une collaboration. Le panneau central et les revers des volets auraient été éxécutés par un premier peintre se mouvant encore dans la tradition du XVe siècle. C'est ce que suggère, en tout cas, le recours à un drapé 'cassé' et 'écrasé' et, parfois aussi, à des plis parallèles. Les figures d'anges musiciens seraient I'œuvre d'un second artiste ayant une conception nettement plus dynamique de la draperie. Ceci l'amènerait à multiplier les plis courbes, rayonnants. En outre, conformément à la nouvelle esthétique du corps héritée de l'art antique, ce second artiste s'efforcerait de rendre visibles les jambes sous l'étoffe.

Si le premier peintre semble essentiellement redevable à David, le deuxième paraît déjà connaître Benson, comme l'indiquent les striures blanches tracées sur la chape de velours rouge de l'ange organiste, un motif typiquement bensonien. On est amené à conclure que ce second peintre devait être plus jeune que son confrère et qu'il se voulait plus moderne. Néanmoins, l'un et l'autre auraient œuvré dans le même atelier. Le répertoire physionomique est identique: le visage de l'ange organiste ressemble à celui de Marie dans le panneau central et dans l'Annonciation. En outre, sur ce même panneau central

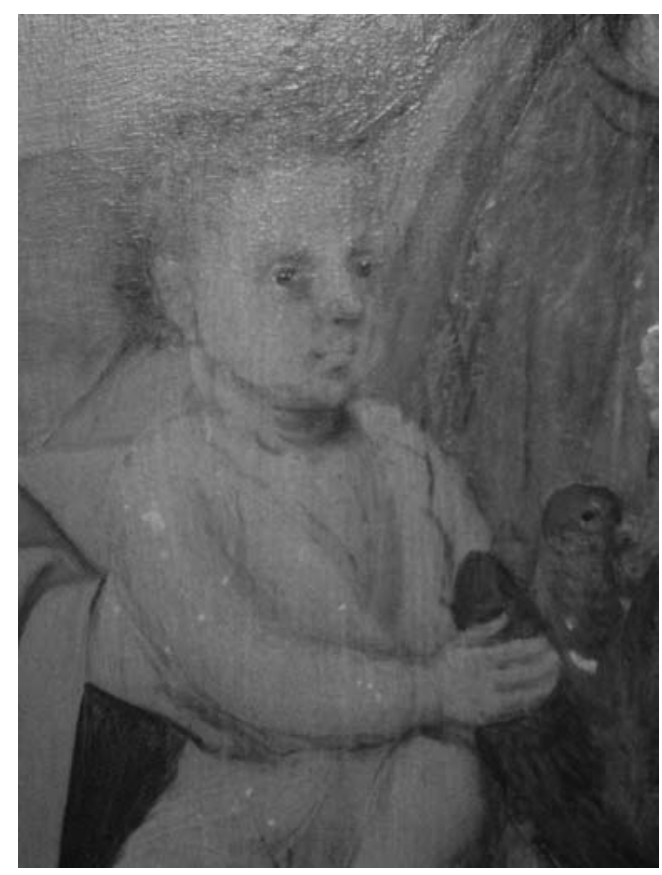

Fig. 11. Anonyme brugeois ( $\mathrm{XV} \mathrm{l}^{\mathrm{e}}$ siècle): Triptyque de la Madone au perroquet, Enfant Jésus (détail dans l'infrarouge). Castrojeriz, San Juan (photo Concha Bengoechea, Burgos) 
et sur le volet gauche, on observe un paysage similaire, scandé par des maisonnettes.

Ne peut-on envisager une autre possiblité? En dépit des différences observées, un seul et même peintre aurait assumé la réalisation de l'ensemble. On a relevé, dans le panneau central et au revers des volets, l'usage de modèles anciens. Ceux-ci auraient-il conditionné le style même de l'exécutant, qui ne les aurait que partiellement actualisés? Dans cette hypothèse, seules les figures d'Anges musiciens des volets, dans lesquelles on peut sans doute voir des inventions de l'artiste, trahiraient son véritable style personnel.

\section{IV.}

La Madone au chanoine Van der Paele, en particulier le groupe central de la Vierge à I'Enfant avec un perroquet, a suscité un grand nombre d'échos dans la peinture flamande de la fin du Moyen Âge et de la Renaissance ${ }^{19}$. II s'agit presque toujours de productions brugeoises: elles peuvent être attribuées notamment au

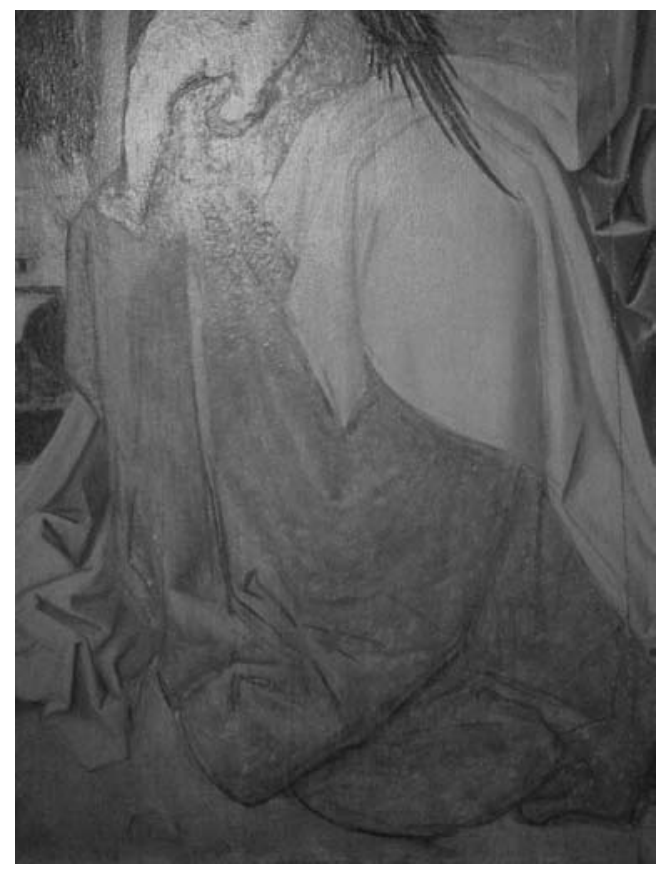

Fig. 12. Anonyme brugeois (XVle siècle): Triptyque de la Madone au perroquet, Madone au perroquet (détail dans l'infrarouge). Castrojeriz, San Juan (photo Concha Bengoechea, Burgos)
Maître des Portraits Baroncelli, au Maître de la Légende de sainte Lucie, à celui de la Légende de sainte Ursule, à Gerard David, au Maître des Madones jouflues, à Adriaen Isenbrant (fig.18) et à Ambrosius Benson (fig. 19). En raison de la présence du modèle dans un espace public - la nef de l'ancienne collégiale Saint-Donatien, devenue cathédrale de Bruges en 1559 -, on aurait pu s'attendre à ce que les copies réalisées dans cette ville aux $X V^{e}$ et $X V l^{e}$ siècles trouvent leur origine dans des études réalisées in situ face à l'original. II n'en est rien. Un détail révélateur indique qu'à une exception près, celle de l'exemplaire de Watervliet aujourd'hui conservé à Anvers, toutes les répétitions connues du panneau eyckien dérivent d'un modèle intermédiaire. Celui-ci se différenciait de l'original eyckien sur un point précis: le dessin de la queue du perroquet.

Jan van Eyck avait jugé bon de dissimuler sous un repli du manteau marial une partie de la queue de l'oiseau et de donner à celle-ci des proportions relativement réduites. Les photographies dans l'infrarouge de la Madone au chanoine Van der Paele permettent de constater que

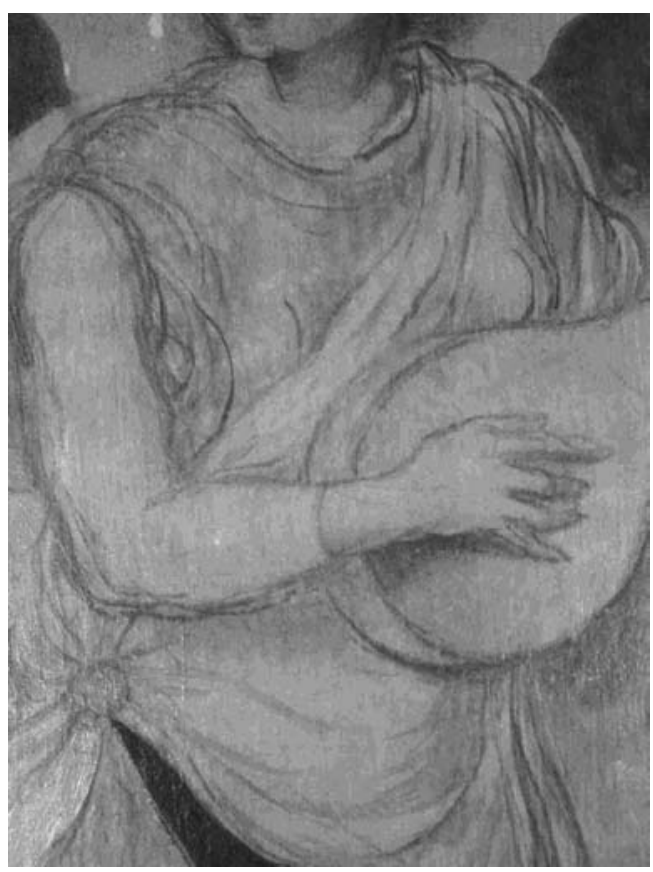

Fig. 13. Anonyme brugeois ( $\mathrm{XV} l^{\mathrm{e}}$ siècle): Triptyque de la Madone au perroquet, Ange au luth (détail dans l'infrarouge). Castrojeriz, San Juan (photo Concha Bengoechea, Burgos) 


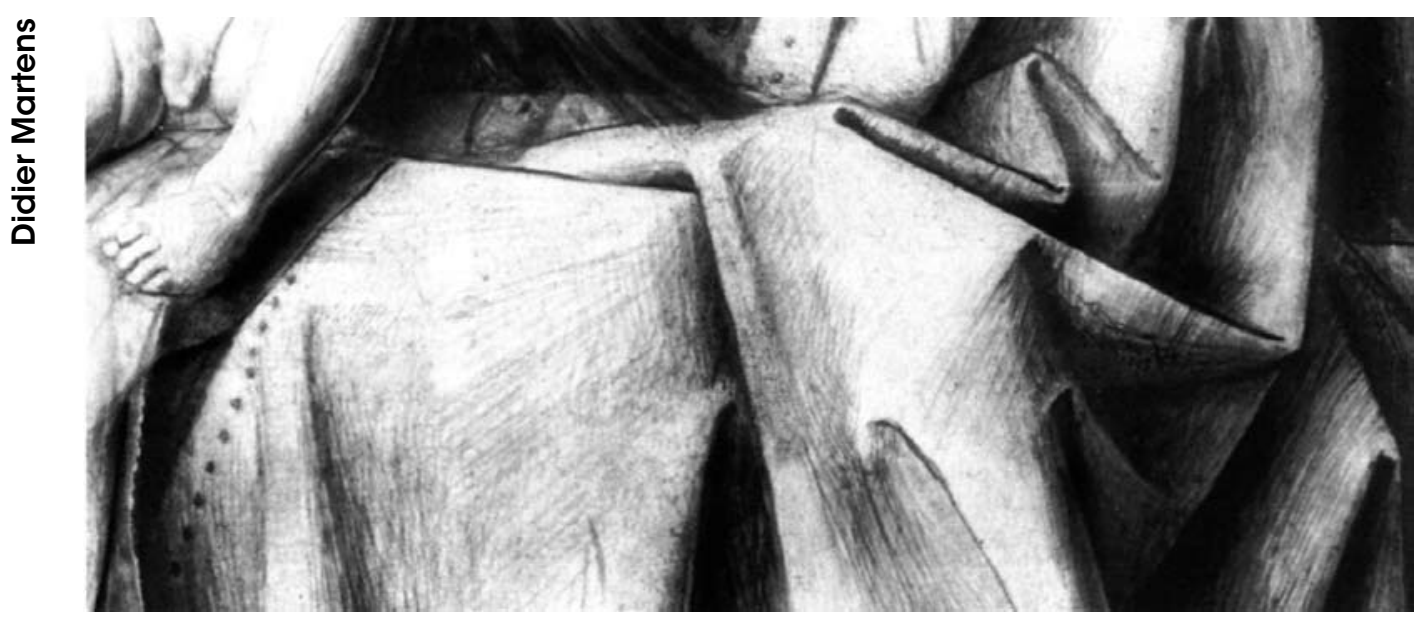

Fig. 14. Jan van Eyck: Madone au chanoine Van der Paele (détail dans l'infrarouge). Bruges, Groeningemuseum (photo Maryan W. Ainsworth, New York)

I'artiste avait adopté cette solution dès le stade du dessin sous-jacent ${ }^{20}$ (fig. 14). Elle ne fut pas du goût de l'un de ses imitateurs, lequel, désireux sans doute $d$ ' 'améliorer' esthétiquement le prestigieux modèle, dota le perroquet d'une longue queue qui se prolonge par-dessus le manteau de la Vierge. C'est cette version interpolée du chef-d'œuvre eyckien qui allait faire école à Bruges. II devait en exister des copies dessinées qui circulèrent parmi les peintres. L'auteur du panneau central du triptyque de Castrojeriz a dû prendre pour modèle l'une de ces copies. Le perroquet qu'il a représenté est en effet pourvu d'une queue se prolongeant par-dessus le manteau marial.

Le fait que les artistes brugeois qui reproduirent la partie centrale de la Madone au chanoine Van der Paele ont eu recours à un modèle intermédaire, plutôt qu'à l'original lui-même, ne doit pas étonner outre mesure. En effet, les copies suscitées aux $X V^{e}$ et $X V l^{e}$ siècles dans les anciens Pays-Bas par l'effigie à mi-corps de Notre-Dame de Grâce de Cambrai, une icône italo-byzantine qu'une pieuse tradition attribuait à saint Luc luimême, ne procèdent pas non plus directement de l'original. II est patent qu'un modèle intermédiaire fut utilisé par les artistes de cette époque $^{21}$. II comportait une interpolation qui se retrouve dans toutes les répétitions de Notre-Dame de Grâce antérieures aux années 1600: I'ourlet rouge du manteau marial dessine, du côté droit, une verticale (fig. 15). Dans l'icône elle-même, c'est une courbe (fig. 16).

Dans les anciens Pays-Bas bourguignons, puis espagnols, ni les autorités ecclésiastiques ni les autorités civiles ne paraissent avoir été enclines à favoriser le travail des copistes in situ. Nous savons par une série de documents d'archives qu'entre 1529 et 1625, le retable de l'Agneau mystique demeurait normalement fermé à clé22. Les volets n'étaient ouverts que lors de visites payantes accompagnées par le clergé. Dans ces conditions, il aurait été difficile pour un artiste de reproduire à la sauvette ne fût-ce qu'une figure du retable. Pour copier l'œuvre, il fallait obtenir une autorisation en bonne et due forme, ce qui nécessitait un puissant protecteur. Ainsi, en 1567, un officier espagnol demanda au chapitre de Saint-Bavon de pouvoir faire copier les panneaux d'Adam et Ėve pour le compte du Commandeur de Sainte-Madeleine ${ }^{23}$. Les chanoines décidèrent de consulter le prévôt du chapitre, le juriste Viglius ab Aytta (1507-1577), qui rendit dans l'affaire un avis prudent, mais favorable, prenant avant tout en considération la qualité du requérant: "Même si j'estime qu'une telle autorisation ne doit pas être accordée à n'importe qui, j'estime aussi qu'on ne doit pas la refuser à des personnes éminentes qui, bénéficiant d'un grand crédit auprès du roi, pourraient, lorsque le besoin s'en fera sentir (?), faire profiter notre église de ses faveurs» ${ }^{24}$. 


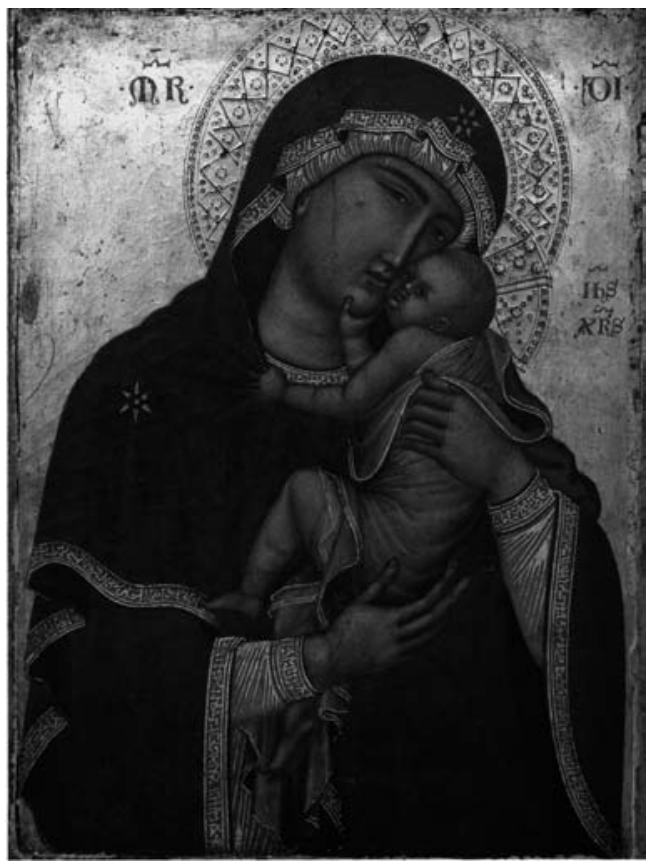

Fig. 15. Anonyme toscan (XIV e siècle): Notre-Dame de Grâce. Cambrai, Cathédrale Notre-Dame de Grâce

Les autorités civiles de Bruxelles étaient peutêtre plus méfiantes encore envers les copistes que le chapitre de Saint-Bavon. La capitale brabançonne s'enorgueillissait de posséder quatre monumentaux panneaux de justice peints par Rogier de le Pasture. Signés et datés 1439, ils étaient exposés dans l'ancienne Gulden Kamer de l'hôtel de ville. Un payement de l'exercice 1499-1500 nous apprend que des copies des panneaux furent détruites par le feu en présence de leurs deux auteurs sur ordre du magistrat de Bruxelles, qui craignait qu'elles puissent être envoyées à l'étranger ${ }^{25}$. On indemnisa toutefois les peintres. La ville semble avoir réussi à empêcher la circulation de répétitions de ses panneaux de justice ${ }^{26}$. En effet, on ne conserve à I'heure actuelle aucune copie fidèle des originaux disparus ou fortement endommagés dans le bombardement de Bruxelles en 169527.

\section{V.}

En tant que modèle, la Madone au chanoine Van der Paele jouissait d'un prestige particulier dans la région de Burgos au milieu du XVIe

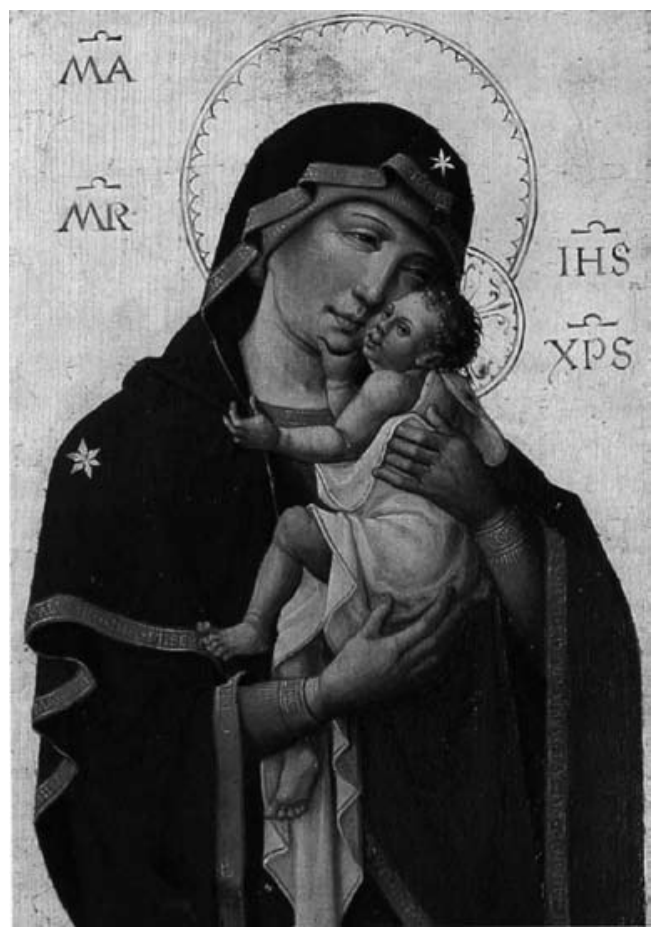

$\frac{0}{\frac{0}{0}}$

Fig. 16. Anonyme flamand (vers 1500): Notre-Dame de Grâce. Lessines, Hôpital Notre-Dame à la Rose (photo KIKIRPA, Bruxelles)

siècle. Outre celui de Castrojeriz, on peut faire état d'un autre triptyque flamand inspiré par le chef-d'œuvre eyckien. II appartient à l'église Saint-Martin de Santa Cruz de Juarros, une localité située à moins de trente kilomètres à l'est de Burgos 28 (fig. 17). Attribuée successivement à Pourbus, puis à Benson, l'œuvre est sans aucun doute brugeoise. Le lien avec Benson est manifeste.

Selon une formule fréquemment attestée à Bruges $^{29}$, le donateur est représenté isolé sur le volet droit, tandis que son saint patron -il s'agit, dans le cas présent, de Jean-Baptiste- occupe le volet gauche. Au centre figure une représentation de la Vierge à l'Enfant, copiée de la Madone au chanoine Van der Paele. Bien entendu, la queue du perroquet est entièrement visible.

Comme dans le triptyque de Castrojeriz, on observe un écart stylistique frappant entre le panneau central et les volets. Si la Vierge à l'Enfant, avec ses plis 'cassés' et 'écrasés', évoque encore 


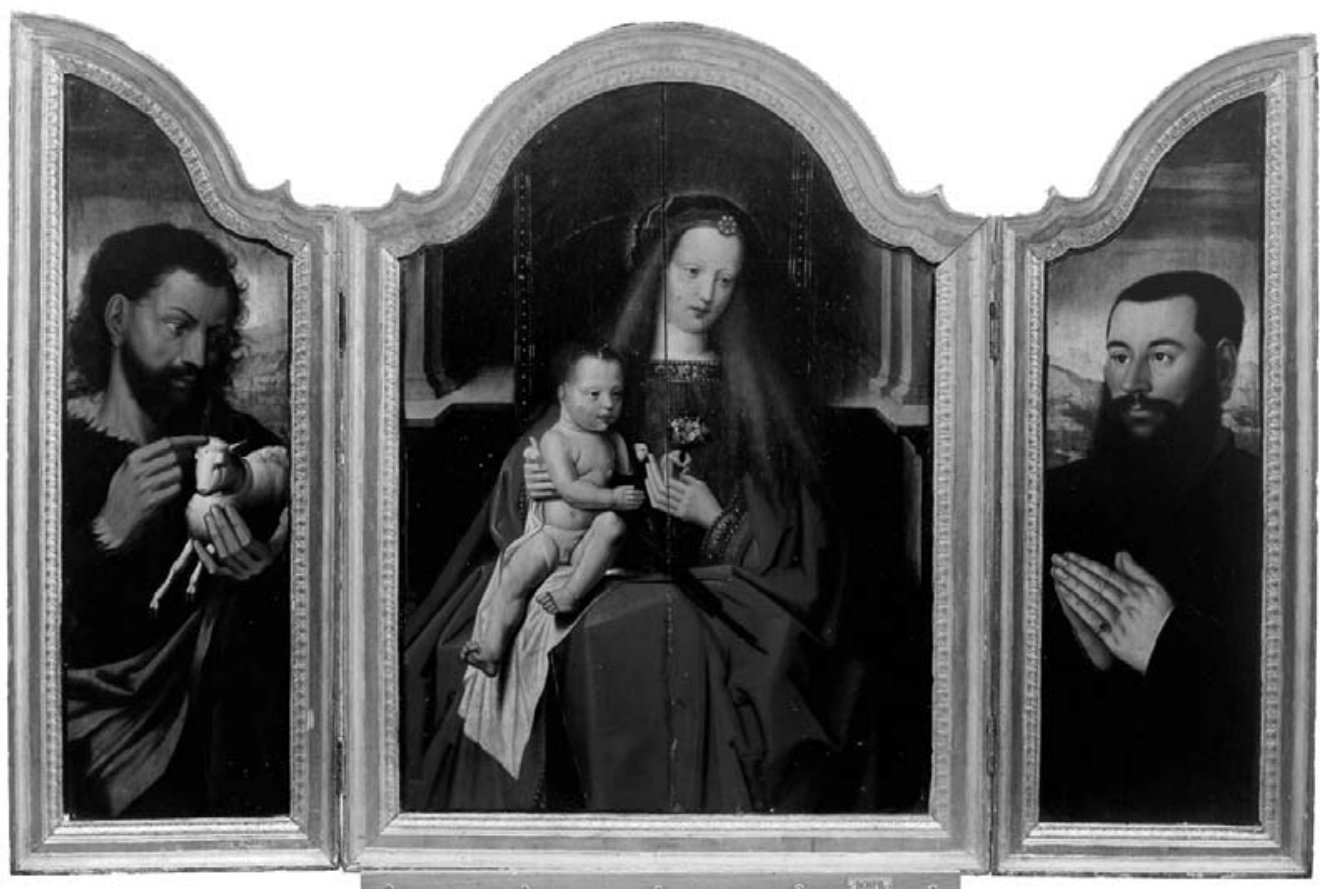

Fig. 17. Anonyme brugeois (XVI e siècle): Triptyque de la Madone au perroquet. Santa Cruz de Juarros, San Martín Obispo (photo Jerónimo Pérez Roca, Grenade)

nettement la tradition des Primitifs du XVe siècle, en revanche, les faces des volets -les revers sont recouverts d'un simple badigeon noir- se signalent par un style plus moderne, caractérisé par un drapé dynamique et des physionomies expressives. À nouveau, la question se pose de savoir comment il convient d'interpréter ces différences. Faut-il envisager une collaboration entre deux artistes et supposer, par exemple, qu'une Vierge à l'Enfant à mi-corps inspirée par la Madone au chanoine Van der Paele aurait été pourvue a posteriori de volets dus à une autre main, dans le but de personnaliser un panneau de dévotion acquis sur le marché libre? Ou bien l'écart perçu s'explique-t-il par le recours à un modèle ancien dans le panneau central, un modèle dont les caractéristiques stylistiques auraient été en grande partie respectées? Dans cette hypothèse, un unique peintre aurait assumé l'exécution de l'ensemble. Son style personnel se dévoilerait seulement dans les volets.

La confrontation des deux triptyques est instructive. Elle met en évidence la diversité du phénomène de la copie flamande des $X V$ e et $X \mathrm{Vl}^{\mathrm{e}}$ siècles, laquelle ne se réduit pas à la simple reproduction de modèles ${ }^{30}$. Si le triptyque de Santa Cruz de Juarros constitue une répétition partielle, en plan rapproché, de la Madone au chanoine Van der Paele, dans le cas de celui de Castrojeriz, on pourrait parler d'un pastiche eyckien: I'Enfant, le perroquet et la main gauche de la Vierge du panneau-épitaphe ont été combinés à une autre source figurée. L'omission délibérée de la figure mariale conçue par Jean van Eyck ne doit pas surprendre outre mesure. En dépit du prestige de la Madone au chanoine Van der Paele -entre 1599 et 1627, elle trôna même sur le maître-autel de Saint-Donatien ${ }^{31}$-, les peintres brugeois qui la reproduirent se permirent souvent des modifications. La réputation acquise dans la seconde moitié du XVI ${ }^{e}$ siècle par l'artiste limbourgeois et le titre de Belgarum splendor qui lui fut alors octroyé ${ }^{32}$ ne pouvaient suffire à contrecarrer l'une des croyances les plus vivaces du moment, celle dans le progrès des arts ${ }^{33}$. II était toujours possible, sinon même souhaitable, 


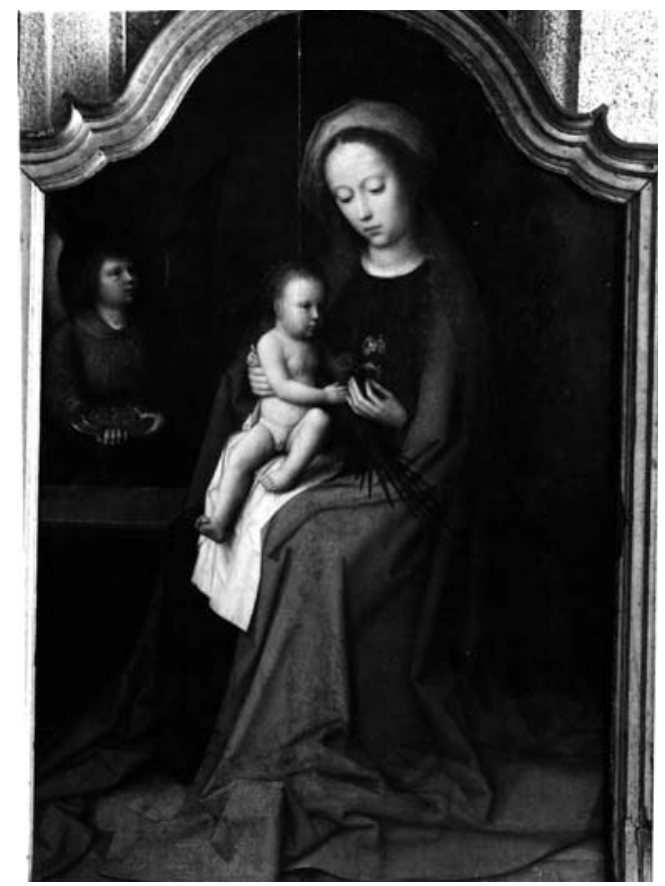

Fig. 18. Adriaen Isenbrant: Triptyque de la Madone au perroquet. Madrid, Monasterio de las Descalzas Reales (photo KIKIRPA, Bruxelles)

d'améliorer un modèle ancien, quel qu'il fût, en le soumettant à une 'critique en acte'34. En outre, il convenait de l'adapter, si nécessaire, à de nouveaux contextes figurés.

Apparemment, plus d'un peintre brugeois de la Renaissance jugeaient que, dans la Madone au chanoine Van der Paele, la figure de la Vierge était moins exemplaire, d'un point de vue esthétique, que celle de l'Enfant. Sa silhouette massive, inscrite dans un triangle quasi équilatéral, a-t-elle déconcerté un public habitué aux représentations mariales d'un Hans Memling ou d'un Gérard David? Toujours est-il que la Madone au perroquet eyckienne a été amincie, non seulement dans le triptyque de Castrojeriz, mais aussi dans trois autres versions brugeoises contemporaines. Ainsi, dans le triptyque attribué à Isenbrant des Descalzas Reales de Madrid ${ }^{35}$ (fig. 18) et dans un panneau similaire qui se trouvait en 1988 dans une collection privée d'Évora ${ }^{36}$, elle est revêtue d'un manteau repris à Gérard David, qui l'a fait paraître plus élancée ${ }^{37}$. De même, dans le panneau du Musée Groeninge de Bruges

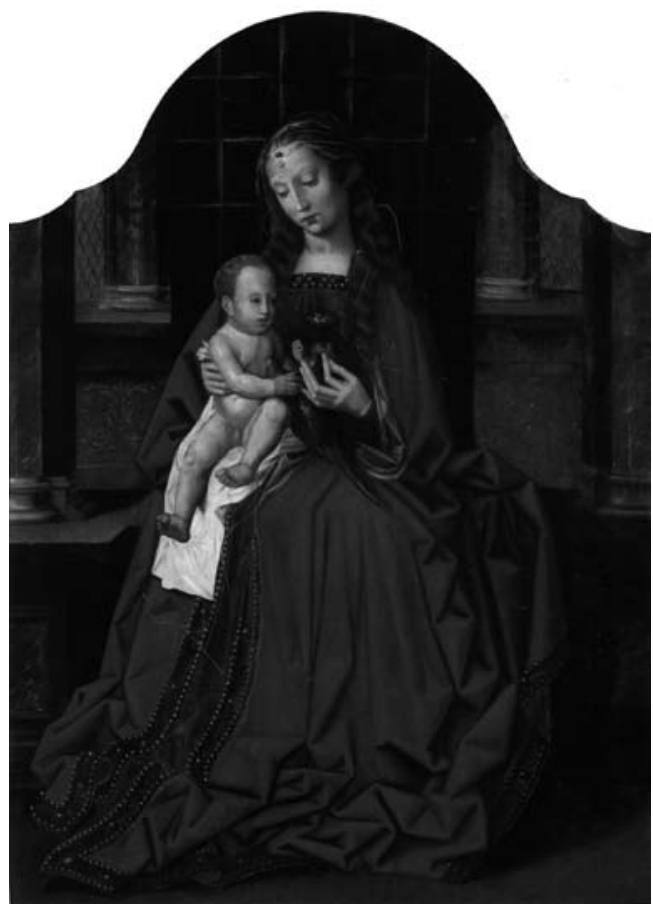

$\frac{0}{\frac{0}{0}}$

Fig. 19. Maître de Castrojeriz: Madone au perroquet. Bruges, Groeningemuseum (photo KIKIRPA, Bruxelles)

attribué à Benson ${ }^{38}$ (fig. 19) -il s'agit en réalité d'une œuvre du 'Maître de Castrojeriz'-, elle arbore un manteau qui n'est pas celui dessiné par Jean van Eyck, même s'il en conserve la couleur, et qui allège considérablement les proportions de la figure ${ }^{39}$.

Dans les trois œuvres qui viennent d'être citées, comme dans le triptyque de Castrojeriz, le visage de Marie est tourné vers la gauche. En revanche, dans la Madone au chanoine Van der Paele, il est dirigé vers la droite. Cette fois, le changement ne relève pas $d$ 'une 'critique en acte' d'un modèle ancien, s'appuyant sur une norme esthétique plus récente, mais correspond plutôt au souci de mettre le groupe marial en conformité avec son nouvel environnement. Jean van Eyck avait décidé de faire regarder le Fils et sa Mère dans la direction de Georges van der Paele agenouillé. Celui-ci occupe, assez logiquement, la partie droite du tableau, le côté gauche, privilégié d'un point de vue hiérarchique ${ }^{40}$, étant réservé au protecteur céleste de la collégiale dont il était prébendier, à savoir saint 
Donatien. La Vierge eyckienne regarde donc vers la droite. Dans le triptyque de Castrojeriz et dans celui des Descalzas Reales, comme dans les panneaux d'Évora et de Bruges qui viennent d'être cités, il n'y a aucun donateur agenouillé qui réclame la bienveillante attention de Marie. Les imitateurs du XVle siècle ont de ce fait jugé préférable de faire pivoter son visage vers la gauche, dans la direction de son Fils. C'est vers lui que, désormais, la Mère de Dieu incline la tête, comme dans la plupart des représentations isolées de la Vierge à l'Enfant.

Le triptyque de Santa Cruz de Juarros comporte, sur le volet droit, une effigie de donateur, lequel s'est en quelque sorte substitué au chanoine. II n'y avait aucune raison de modifier I'orientation du visage marial eyckien. La Madone du triptyque regarde donc vers la droite.

\section{VI.}

Les triptyques de Castrojeriz et de Santa Cruz de Juarros ont dû quitter Bruges peu après leur achèvement, dans les années 1530-1540. Ils attestent la pénétration, au cœur de la Castille, d'une image flamande vieille alors d'une centaine d'années. Comment expliquer ce succès? Une hypothèse peut être avancée. Au milieu $\mathrm{du} X \mathrm{XI}{ }^{\text {e }}$ siècle, le panneau eyckien conservé à Saint-Donatien devait avoir acquis, aux yeux des visiteurs étrangers, et en particulier des Espa- gnols, le statut d'un véritable souvenir de Bruges. La présence du perroquet, motif exotique plutôt exceptionnel dans la peinture religieuse, aura sans doute contribué à établir la célébrité du tableau. À l'instar des touristes actuels qui aiment à se faire photographier devant la Tour Eiffel ou Buckingham Palace, le commanditaire du triptyque de Santa Cruz de Juarros a pu désirer associer son effigie personnelle à celle d'un monument brugeois célèbre: la Madone au chanoine Van der Paele. Le fait qu'il a posé pour un peintre proche de Benson indique en tout cas qu'il a effectivement séjourné à Bruges.

Si le triptyque de Juarros reproduit assez fidèlement le groupe central du panneau eyckien, en revanche, le triptyque de Castrojeriz propose ce même monument brugeois sous une forme radicalement modernisée. Cette modernisation concerne non seulement les proportions de la figure mariale, mais aussi son environnement. L'intérieur d'église roman conçu par Jan van Eyck a fait place, en effet, à un espace naturel ouvert, conformément à la nouvelle mode du paysage cultivée par Gérard David ${ }^{41}$. La remarquable flexibilité de l'offre des peintres brugeois de la Renaissance, lesquels étaient capables de subtilement doser, dans une image, tradition et modernité, explique sans doute aussi, pour une part, leur succès international, jusqu'en Castille.

\section{NOTES}

* C'est un agréable devoir de remercier ici Marta Negro Cobo et René-Jesús Payo Hernanz (Burgos), qui ont toujours eu à cœur de partager avec moi leur connaissance du patrimoine artistique castillan et qui m'ont efficacement aidé à mener à bien la présente étude. Je tiens aussi à exprimer ma reconnaissance à Claire Baisier (Anvers), Carmen García-Frias Checa (Madrid), Géraldine Patigny (Bruxelles) et Ingrid Staes (Anvers), ainsi qu'à ma collègue Ghislaine Viré (Bruxelles). Comme de coutume, j'ai bénéficié de I'hospitalité du Centre d'étude des
Primitifs flamands (Bruxelles). Merci à Dominique Deneffe, à Bart Fransen, à Famke Peters et à Cyriel Stroo pour leur aimable accueil. Enfin, mon texte a été relu par Bruno Bernaerts, Jacques de Landsberg, Georges Hupin et Monique Renault.

${ }^{1}$ Marta NEGRO COBO, "Los mercaderes de Castrojeriz en los siglos XV y XVI», dans: De Castrojeriz a Brujas. Mecenazgo en la iglesia de San Juan, Castrojeriz, 2010, pp. 25-26.

2 Voir, sur cet ensemble, Didier MARTENS, «El retablo de los Gallo, un ejemplo de mestizaje cultural hispano-flamenco», dans: De Castrojeriz a Brujas, op. cit., pp. 155-220; idem,
Peinture flamande et goût ibérique aux $X V^{\text {e }}$ et XVI e siècles, Bruxelles, 2010, pp. 263-297.

${ }^{3}$ Castrojeriz, San Juan; huile sur bois; triptyque, fermé (avec cadre): 69 x $58 \mathrm{~cm}$, ouvert (avec cadre): $69 \times 116$ $\mathrm{cm}$. La présence actuelle de I'œuvre à San Juan m'a été confirmée par Marta Negro Cobo, Museo de Burgos (courriel à l'auteur, 6 juin 2012).

${ }^{4}$ Voir, à ce sujet, D. MARTENS, Peinture flamande et goût ibérique, op. cit., pp. 13-23.

5 Voir, sur cette œuvre, Jochen SANDER, Die Entdeckung der Kunst. Niederländische Kunst des 15. und des 16. Jahrhunderts in Frankfurt (cat. 
d'exp.), Francfort-sur-le-Main, Städelsches Kunstinstitut und Städtische Galerie, 1995-1996, pp. 14-16 et $n^{\circ} 1$.

${ }^{6}$ Voir, sur cette œuvre, Hans VLIEGHE, Saints, /I (Corpus rubenianum Ludwig Burchard, 8), Bruxelles, 1973, n's 146-149.

${ }^{7}$ Voir, sur cette œuvre, Uta NEIDHARDT, notice, Das Geheimnis des Jan van Eyck. Die frühen niederländischen Zeichnungen und Gemälde in Dresden (cat. d'exp.), Dresde, Residenzschloss, 2005, n67.

${ }^{8}$ Au stade du dessin sous-jacent, l'ange organiste était coiffé d'un diadème surmonté d'une croix. Ce motif n'a pas été retenu au stade de l'exécution picturale.

${ }^{9}$ Voir, sur cette œuvre, Hugo VAN DER VELDEN, «Diptych Altarpieces and the Principle of Dextrality», dans: Unfolding the Netherlandish Diptych. Essays in Context, Washington, 2006, pp. 139-142.

${ }^{10}$ VII Centenario de la catedral de Burgos. Exposición de Arte retrospectivo. Catálogo general, Burgos, 1926, p. 29, n 1116: "Tríptico cuya tabla central es copia de un Gerard David perdido. Los ángeles de las laterales italianizados son obra de copista. Representa a la Virgen ofreciendo al Niño un pájaro y flores. Siglo XVI. - Parr. de Castrojeriz». Voir depuis lors, sur ce triptyque, Luciano HUIDOBRO SERNA / José ALONSO, El camino de Santiago a su paso por Castrojeriz, Burgos, 1965, p. 40; Agustín LÁZARO LÓPEZ, notice, dans: Las Edades del Hombre: la música en la Iglesia de Castilla y León (cat. d'exp.), Léon, Catedral, 1991-1992, n 110; Ángel RUIZ GARRASTACHO, Castrojeriz, Castrojeriz, 1992, p. 77; 2001, p. 66; René-Jesús PAYO HERNÁNZ, Catálogo de pintura de la Villa de Castrojeriz, Castrojeriz, 1999, n² 2

${ }^{11}$ R. J. PAYO HERNÁNZ, op. cit., p.46.

12 R. J. PAYO HERNÁNZ, op. cit., p. 50 .

${ }^{13}$ Voir, sur cette œuvre, Maryan W. AINSWORTH, notice, dans: From Van Eyck to Bruegel. Early Netherlandish Painting in the Metropolitan Museum of Art (cat. d'exp.), New York, Metropolitan Museum of Art, 1998-1999, $n^{\circ} 78$.
${ }^{14}$ Voir, sur cette œuvre, Georges MARLIER, «Un triptyque inédit d'Ambrosius Benson: Saint Jacques à la bataille de Clavijo», dans: Revue belge $d$ 'Archéologie et d'Histoire de l'Art, 34, 1965, pp. 75-84.

15 Voir, sur ces œuvres, Friso LAMMERTSE, notice, dans: Van Eyck to Bruegel 1400-1550. Dutch and Flemish Painting in the Collection of the Museum Boymans-van Beuningen (cat.d'exp.), Rotterdam, Museum Boymans-van Beuningen, 1994, n 38.

$$
{ }^{16} \text { A. LÁZARO LÓPEZ, op. cit, p. }
$$
182.

17 Voir, sur cette œuvre, Aquilin JANSSENS DE BISTHOVEN / Marguerite BAES-DONDEYNE / Dirk DE VOS, Musée Communal des Beaux-Arts (Musée Groeninge) (Corpus de la peinture des anciens Pays-Bas méridionaux et de la Principauté de Liège au XV siècle, 1), Bruxelles, 1983, n 9.

${ }^{18}$ A. LÁZARO LÓPEZ, op. cit., pp. 181-182; R.J. PAYO HERNANZ, op. cit., p. 49.

19 Voir, à ce sujet, A. JANSSENS DE BISTHOVEN / M. BAES-DONDEYNE / D. DE VOS, op. cit., pp. 210-213; Paul VANDENBROECK, Koninklijk Museum voor Schone Kunsten Antwerpen. Catalogus schilderkunst 14e-15e eeuw, Anvers, 1985, pp. 178-180; Maryan W. AINSWORTH, Gerard David. Purity of Vision in an Age of Transition, New York, 1998, pp. 163-165; Didier MARTENS, «Échos de la Madone au chanoine Van der Paele, en particulier dans I'œuvre du Maître brugeois de la Légende de sainte Ursule», dans: Mélanges offerts à Claire Dickstein-Bernard (Annales de la Société royale d'Archéologie de Bruxelles, 63), Bruxelles, 1999, pp. 195-222; Hugo VAN DER VELDEN, The Donor's Image. Gerard Loyet and the Votive Portraits of Charles the Bold, Turnhout, 2000, pp. 84-87, 124.

${ }^{20}$ Voir le document reproduit dans Maryan W. AINSWORTH, "Revelations about Jan van Eyck's Virgin and Child with Saints Donatian and George and the Canon Van der Paele», dans: Le dessin sous-jacent et la technologie dans la peinture. Colloque XIV, 13-15 septembre 2001, Bruges-Rotterdam, Louvain / Paris / Dudley, 2003, p. 279, fig. 7 .
${ }^{21}$ Voir, à ce sujet, Didier MARTENS, notice, dans: Séminaire de Tournai: Histoire. Bâtiments. Collections, Louvain, 2008, pp. 192-193.

${ }^{22}$ Voir, à ce sujet, Elisabeth DHANENS, De Vijd-Borluut fundatie en het Lam Gods retabel (Mededelingen van de Koninklijke Academie voor Wetenschappen, Letteren en Schone Kunsten van België. Klasse der Schone Kunsten, 38, 2), Bruxelles, 1976, pp. 21, 46-48; Jan VAN DER STOCK, "Canon in Context. Consumption of Early Netherlandish Images in the Fifteenth and the First Half of the Sixteenth Centuries», dans: Rogier van der Weyden in Context (Underdrawing and Technology in Painting. Symposium, 17), Paris / Louvain / Walpole, 2012, p. 7.

${ }^{23}$ Voir, à ce sujet, Jozef DUVERGER, «Kopieën van het Lam Godsretabel van Hubrecht en Jan van Eyck», dans: Bulletin des Musées royaux des Beaux-Arts de Belgique, 3, 1954, pp. $60,67$.

24 J. DUVERGER, op. cit., p. 63: «Resolvitque eadem Reverentia sua [Viglius] in hunc modum: quod etsi non cuilibet eam facultatem concedendam arbitror, tamen proceribus viris quique cum apud regem gratia poleant tum ecclesiam nostram suo favore ubi usus niterit juvare possint denegandum hoc non arbitror».

25 Elisabeth DHANENS, Rogier van der Weyden. Revisie van de documenten (Verhandelingen van de Koninklijke Academie voor Wetenschappen, Letteren en Schone Kunsten van België. Klasse der Schone Kunsten, 57, 1995, n 59), Bruxelles, 1995, pp. 124-125: "Betaelt twee scilders, die de vier stucken van der scilderyen opter gulden camere in der stadthuys constelick geconterfayt hadden en de voirs. patroenen in ander landen souden hebben moegen seynden, dwelc in der wethouderen camere in hueren presentien, mits sekeren redenen den heeren daertoe porrende, verbrant was, gegeven ter ordinancien van der wet uuyt gratien 4 Rgld [Rijnsgulden]».

${ }^{26}$ Voir, dans le même sens, J. DUVERGER, qui avait déjà rapproché les deux textes cités aux notes 24 et 25 (op. cit., p. 55). 
${ }^{27}$ Voir, sur ces panneaux et leurs échos, Dirk DE VOS, Rogier van der Weyden. Het volledige œuvre, Anvers, 1999, pp. 57-60, 345-354.

${ }^{28}$ Ibeas de Juarros, Santa Cruz de Juarros (Burgos), San Martín Obispo; huile sur bois; triptyque chantourné, fermé (avec cadre): $77 \mathrm{~cm}(60 \mathrm{~cm}) \mathrm{x}$ $58,5 \mathrm{~cm}$, ouvert (avec cadre): $77 \mathrm{~cm}$ (60 $\mathrm{cm}) \times 117 \mathrm{~cm}$. Actuellement en dépôt à Burgos, Museo del Retablo (courriel à I'auteur de Marta Negro Cobo, Museo de Burgos, 6 juin 2012). Voir, sur cette œuvre, VII Centenario de la catedral de Burgos, op. cit., p. 20, n²01; María, peregrina de la Fe (cat. d'exp.), Burgos, Casa de la Iglesia, 1988, nº 11.

${ }^{29}$ Voir, à ce sujet, Didier MARTENS / Barbara KISS, «Le triptyque de Ontaneda aux Musées royaux des BeauxArts de Bruxelles: typologie, attribution et fortune critique», dans: Revue belge d'Archéologie et d'Histoire de l'Art, 72, 2003, pp. 117-121.

${ }^{30}$ Voir, sur la copie flamande des $X V^{e}$ et $X V^{e}$ siècles, récemment, Hélène MUND, "Original, copie et influence: une histoire complexe», dans: Rogier van der Weyden 1400-1464. Maitre des Passions (cat. d'exp.), Louvain, Museum M, 2009, pp. 186-2005; Ariane MENSGER, «Die exakte Kopie. Oder: die Geburt des Künstlers im Zeitalter seiner Reproduzierbarkeit», dans: Het beeld van de kunstenaar in de vroegmoderne Nederlanden (Nederlands kunsthistorisch Jaarboek, 59), Zwolle, 2010, pp. 195-221; Stephan KEMPERDICK, «Von der Vorlage zum Kunstwerk. Rogier van der Weydens Grosse Kreuzabzahme», dans: Original - Kopie - Zitat.
Kunstwerke des Mittelalters und der frühen Neuzeit: Wege der Aneignung - Formen der Überlieferung (Veröffentlichungen des Zentralinstituts für Kunstgeschichte in München, 26), Passau, 2010, pp. 207-230.

${ }^{31}$ Voir, à ce sujet, A. JANSSENS DE BISTHOVEN / M. BAES-DONDEYNE / D. DE VOS, op. cit., pp. 205, 225.

32 Voir, à ce sujet, Ariane MENSGER, «Jan van Eyck Belgarum Splendor und der Anfang einer niederländischen Geschichte der Kunst», dans: Pantheon, 58, 2000, pp. 44-53.

33 Voir, à ce sujet, notamment Ernst GOMBRICH, Kunst und Fortschritt. Wirkung und Wandlung einer Idee, Cologne, 1978; Olga HAZAN, Le mythe du progrès artistique. Étude critique d'un concept fondateur du discours sur l'art depuis la Renaissance, Montréal, 1998.

${ }^{34}$ Voir, à ce sujet, Didier MARTENS, «Du Saint Luc peignant la Vierge à la copie des maîtres: la 'norme en acte' dans la peinture flamande des $\mathrm{XV}^{\mathrm{V}}$ et $\mathrm{XV} \mathrm{V}^{\mathrm{e}}$ siècles», dans: Revue belge d'Archéologie et d'Histoire de l'Art, 74, 2005, pp. 3-50.

${ }^{35}$ Voir, sur cette œuvre, Elisa BERMEJO MARTíNEZ, "Colecciones del Patrimonio Nacional. Pintura IX: Primitivos flamencos (1)», dans: Reales Sitios, 9, 1972, n 33, p. 42; Ana GARCÍA SANZ / María Leticia SÁNCHEZ HERNÁNDEZ, Guía Reales Monasterios de Madrid: Las Descalzas y La Encarnación, Madrid, 2008, pp. 39-41.

${ }^{36}$ Voir, sur cette œuvre, Iconografia mariana no Alto Alentejo (cat. d'exp.),
Évora, Museu de Évora, 1988, n²6; D. MARTENS, op. cit., 1999, p. 203.

${ }^{37}$ Voir, sur ce modèle, attesté notamment par un Repos durant la Fuite en Égypte de David conservé à New York, M. W. AINSWORTH, op. cit., 1998, pp. 283-286.

${ }^{38}$ Voir, sur cette œuvre, Dirk DE VOS, Stedelijke Musea Brugge. Catalogus schilderijen 15de en 16de eeuw, Bruges, 1979, pp. 80-81; D. MARTENS, «El retablo de los Gallo, un ejemplo de mestizaje cultural hispano-flamenco», dans: De Castrojeriz a Brujas, op. cit., pp. 211-215.

39 Je n'ai pu jusqu'à présent identifié l'origine du modèle utilisé par le Maître de Castrojeriz pour ce manteau, mais il semble clair qu'il s'agit d'un modèle ancien, remontant au XV ou au début du XVle siècle.

${ }^{40}$ Voir, à ce sujet, Marie-Léopoldine LIEVENS-DE WAEGH, "Quelques symboles fondamentaux chez les 'Primitifs flamands'», dans: Miscellanea Henri Pauwels (Bulletin des Musées royaux des Beaux-Arts de Belgique, 3840), Bruxelles, 1991, pp. 149-152; eadem, «Le langage de l'image chez Hans Memlinc. En marge de l'exposition de Bruges (1 ìre partie)», dans: Revue des Archéologues et Historiens d'Art de Louvain, 27, 1994, pp. 62-65.

${ }^{41}$ Voir, à ce sujet, Gerard David y el paisaje flamenco (Contextos de la colección permanente, 15) (cat. d'exp.), Madrid, Museo Thyssen-Bornemisza, 2004. 